Two-step strain analysis of self-assembled InAs/GaAs quantum dots

This article has been downloaded from IOPscience. Please scroll down to see the full text article.

2006 Semicond. Sci. Technol. 21626

(http://iopscience.iop.org/0268-1242/21/5/010)

The Table of Contents and more related content is available

Download details:

IP Address: 140.112.113.225

The article was downloaded on 17/12/2008 at 09:02

Please note that terms and conditions apply. 


\title{
Two-step strain analysis of self-assembled InAs/GaAs quantum dots
}

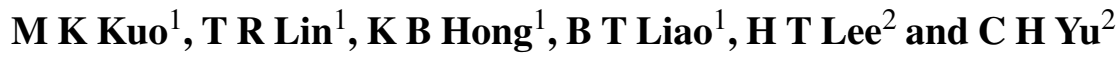 \\ ${ }^{1}$ Institute of Applied Mechanics, National Taiwan University, No 1, Sec 4, Roosevelt Road, \\ Taipei 10672, Taiwan, Republic of China \\ ${ }^{2}$ Chung-Shan Institute of Science and Technology, Taoyuan 325, Taiwan, Republic of China \\ E-mail: mkkuo@ntu.edu.tw
}

Received 8 August 2005, in final form 19 February 2006

Published 24 March 2006

Online at stacks.iop.org/SST/21/626

\begin{abstract}
Strain effects on optical properties of self-assembled InAs/GaAs quantum dots grown by epitaxy are investigated. Since a capping layer is added after the self-assembly process of the quantum dots, it might be reasonable to assume that the capping layer neither experiences nor affects the induced deformation of quantum dots during the self-assembly process. A new two-step model is proposed to analyse the three-dimensional induced strain fields of quantum dots. The model is based on the theory of linear elasticity and takes into account the sequence of the fabrication process of quantum dots. In the first step, the heterostructure system of quantum dots without the capping layer is considered. The mismatch of lattice constants between the wetting layer and the substrate is the driving source for the induced elastic strain. The strain field obtained in the first step is then treated as an initial strain for the whole heterostructure system, with the capping layer, in the second step. The strain from the two-step analysis is then incorporated into a steady-state effective-mass Schrödinger equation. The energy levels as well as the wavefunctions of both the electron and the hole are calculated. The numerical results show that the strain field from this new two-step model is significantly different from models where the sequence of the fabrication process is completely omitted. The calculated optical wavelength from this new model agrees well with previous experimental photoluminescence data from other studies. It seems reasonable to conclude that the proposed two-step strain analysis is crucial for future optical analysis and applications.
\end{abstract}

\section{Introduction}

Due to their three-dimensional quantum confinements, quantum dots (QDs) possess several interesting characteristics, such as delta-function distributions of the density of states, discrete energy levels and 'atom-like' electronic states. These characteristics and their potential applications in optoelectronics have caused QDs to attract substantial attention recently [1,2]. The optoelectronic efficiency of QDs is strongly related to the distribution density of dots in a QD array. Self-assembled QDs (SAQDs) formed by strained epitaxy have shown promising results in producing dense and large QD arrays. SAQD formation is commonly observed in large mismatch epitaxy of chemically similar materials. For example, the Stranski-Krastanow (SK) growth of InAs on
GaAs first involves the growth of a 'wetting layer' of 1-2 monolayer thick followed by a spontaneous coherent island formation [1-3]. SAQDs may often be buried by a further deposition of other materials, usually the same as the substrate, to protect the quantum dots.

The strain fields inside and in the neighbourhood of SAQDs would strongly affect their electronic as well as optoelectronic properties [4]. For III-V semiconductor materials (such as InAs and GaAs), there are two predominant strain effects on the optoelectronic properties, namely the induced changes of levels of the conduction band and of the valence band. The conduction band is affected by the hydrostatic strains, often referred to as dilatation or trace of strain tensors. The valence levels could be altered with both hydrostatic and biaxial strains. 
To understand the strain effects on the optical properties of QDs, accurate determination of the induced strain fields in the dots and the surrounding matrix is certainly necessary. Methods of performing strain analysis of QDs can be catalogued into three groups: (i) theory of inclusions based on the analytical solution of elasticity [5, 6], (ii) continuum theory together with finite element methods (FEM) [7-13] and (iii) atomistic modelling $[14,15]$. The theory of inclusions provides integral expressions for the strain fields, which can even be integrated in closed forms for QDs with some special shapes, e.g. cylindrical or spherical QDs. On the other hand, the interactions between the QDs and the surrounding materials may not be fully encountered in these approaches. Atomistic models might be more reasonable among all these three groups, at least theoretically, to model systems in nano-scale provided that accurate interatomic potentials are available. Nevertheless, atomistic modelling would require a huge computing capacity to model QDs and the surrounding matrix together. FEM is a very versatile and effective numerical method. It can easily accommodate various theories to model QDs to different levels of complexity as one would desire.

In this paper, a novel two-step model based on the theory of linear elasticity is developed to evaluate the strain field in the InAs/GaAs SAQD nanostructure. The model takes the sequence of the fabrication process of the self-assembled QDs into account. The mismatches of lattice constants in the heterostructures induce the strain fields which are calculated with the aid of a finite element package FEMLAB. The carrier confinement potential in the three-dimensional steady-state Schrödinger equation is then modified by strain distribution. The equation is again solved by FEMLAB. The solutions consist of energy levels and wavefunction spectra in SAQD nanostructures. The energies of interband optical transitions are obtained and compared against experimental data from other studies as well as numerical results from two other continuum models. Finally, strain effects on optical properties in pyramidal InAs/GaAs quantum dot are discussed.

\section{Statement of the problem}

The fabrication of SAQDs via SK growth can be viewed as a sequence of three different stages, namely, epitaxy, QD formation and capping layer deposition stages. For example, a thin wetting layer of InAs is first grown on a (00 01 ) GaAs substrate during heteroepitaxy. A spontaneous coherent island formation is then followed to form SAQDs. Finally, the quantum-dot islands are covered by a deposition of an additional material; usually it is the same material as the substrate. To elaborate on the proposed two-step strain analysis approach, a single square-based pyramidal selfassembled InAs QD buried in the GaAs matrix, as depicted in figure 1 , for simplicity, is considered. Two different sizes of quantum-dot islands $16 \times 3 \mathrm{~nm}$ and $16 \times 6 \mathrm{~nm}$ (width $\times$ height), respectively, are considered. Each size possesses the cases of buried and surface QDs, namely, QD with/without the capping layer, respectively. The thicknesses of the substrate and the capping layer are $50 \mathrm{~nm}$ and $20 \mathrm{~nm}$, respectively.

Analysis of strain effects on optical properties of SAQDs is divided into two parts in this paper. First, a novel two-

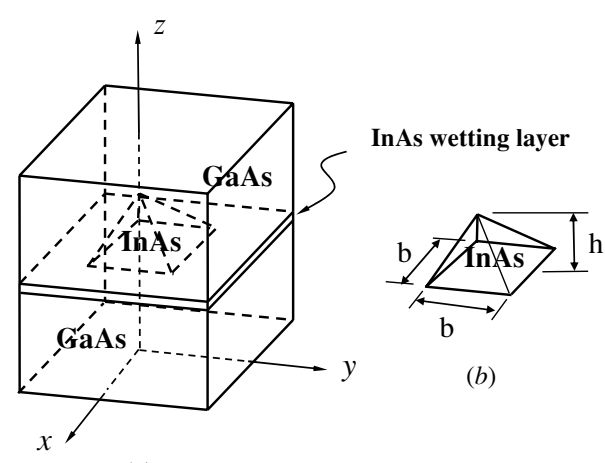

(a)

Figure 1. Schematics and geometries of $(a)$ the buried InAs/GaAs QD nanostructure and $(b)$ the InAs quantum-dot island; $b$ and $h$ are the width and height of the island, respectively.

step model based on the theory of linear elasticity is proposed to determine strain distribution in InAs/GaAs nanostructures via finite element calculation. Second, the strain effects on the carrier confinement potential in nanostructures are incorporated with the aid of Pikus-Bir Hamiltonian and Luttinger-Kohn formalism. The resulting three-dimensional steady-state Schrödinger equation is then solved numerically to obtain the energy levels and wavefunction spectra in SAQD.

\section{Strain field}

Epitaxially grown semiconductor heterostructures, such as SAQDs, often consist of more than one material with different lattice constants. The mismatch of lattice constants gives rise to strain fields in QD nanostructures, which will affect the optical properties of quantum dots [16]. In-plane lattice mismatch parameters are defined as usual:

$$
\left(\varepsilon_{0}\right)_{x x}=\left(\varepsilon_{0}\right)_{y y}=\frac{a_{\mathrm{s}}-a_{\mathrm{d}}}{a_{\mathrm{d}}},
$$

where $a_{\mathrm{s}}$ and $a_{\mathrm{d}}$ are the lattice constants of the substrate (GaAs) and the dot (InAs) materials, respectively, which will be taken as 0.565 and $0.605 \mathrm{~nm}$ in the numerical computation of this paper. Obviously, in this example, the lattice constant of the dot material (InAs) exceeds that of the substrate material (GaAs). Hence, it is expected that InAs will experience compression from GaAs, while GaAs will experience tension from InAs.

Note that the lattice mismatch parameters defined in equation (1) are not yet the complete strain fields in the quantum-dot island. In fact, lattice mismatches will induce further elastic deformation in the whole nanostructure system, in both the substrate and the island, to ensure the equilibrium of the corresponding stresses. In this paper, the lattice mismatch parameter will be treated as in-plane initial strain in the latter analysis to find the complete strain fields $[12,13]$.

It is a common belief that in-plane initial strains in the epitaxy process will certainly accompany out-of-plane strains. On the other hand, out-of-plane initial strains have never yet been as conclusive as their in-plane counterparts [13]. In this paper, out-of-plane initial strains are considered as if the in-plane initial strains were strained elastically and were 
in plane-stress states, which then, owing to Poisson's effect, lead to

$$
\left(\varepsilon_{0}\right)_{z z}=-\frac{2 C_{12}}{C_{11}}\left(\varepsilon_{0}\right)_{x x} .
$$

Here, both GaAs and InAs are treated as cubic materials, each with three independent elastic moduli, namely, $C_{11}, C_{12}$ and $C_{44}$. The numerical values of elastic moduli used in this paper are taken from [17].

In this paper, the parameters $\left(\varepsilon_{0}\right)_{x x},\left(\varepsilon_{0}\right)_{y y}$ and $\left(\varepsilon_{0}\right)_{z z}$ are regarded as the initial normal strains in the $x$-, $y$ - and $z$-directions, respectively. These initial strains in the wetting layer and the island will induce further strain fields in the whole SAQD system. Thus they will be used as inputs in the latter finite element analysis.

According to the theory of linear elasticity, the relationship among stresses $\sigma_{i j}$, total strains $\varepsilon_{k l}$ and initial strains can be expressed as

$$
\sigma_{i j}=C_{i j k l}\left[\varepsilon_{k l}-\left(\varepsilon_{0}\right)_{k l}\right] \quad i, j, k, l=x, y, z,
$$

where $C_{i j k l}$ is the component of the fourth-order tensor of elastic moduli, and $\left(\varepsilon_{0}\right)_{k l}$ is the initial strain tensors described in equations (1) and (2) with

$$
\left(\varepsilon_{0}\right)_{k \ell}=0, \text { for } k \neq \ell .
$$

The contracted notation has been used for elastic moduli, namely $x x \rightarrow 1, y y \rightarrow 2, z z \rightarrow 3, x y \rightarrow 6, y z \rightarrow 4$, $z x \rightarrow 5$.

The linear elastic boundary value problem, arising from the mismatch in lattice constants between the island and substrate materials, is analysed using the finite element package. Appropriate boundary conditions are necessary in the analysis: all the nodes of the $x$ - and $y$-outer surfaces are fixed against displacement in the normal direction due to periodic symmetric argument. The bottom outer surface is fixed against displacement in the $z$-direction to avoid rigid body shift while the upper surface is kept traction free. The compatibility of displacement across the interface of island/substrate is satisfied automatically in the finite element formulation with displacement field used as unknowns.

During the QD fabrication process, the wetting layer grows on the substrate up to a critical thickness, followed by a spontaneous quantum-dot island formation. Finally, the system is buried by an additional deposition of a capping layer, as shown in figure 1. Since the capping layer is deposited after the self-assembly process of islands, the capping layer has no chance to experience or to affect the induced deformation of islands during the self-assembly process. Here, a two-step model is developed to investigate the strain fields induced by the mismatch of lattice constants in the heterogeneous nanostructure. Results obtained from the other two models, based on the continuum theory, are also presented and compared to examine the merits of the proposed two-step model.

- Model I (conventional model). The capping layer together with the island, the wetting layer and the substrate is assumed to respond and deform as a single body. The driving source of deformation is of course the mismatch of lattice constants. This is the conventional model used in almost all the previous studies $[7,8,13]$.
- Model II (proposed two-step model). A two-step strain analysis is developed in this paper. Since the capping layer is added after the SAQD formation, it is hence excluded in the first step of the strain analysis of the model. Thus, in the first step of the strain analysis, the QD heterostructure is considered to consist only the island, the wetting layer and the substrate but not the capping layer. As in model I, the mismatch of lattice constants of the heterostructure is the driving source for the induced strain in this step. The resulted total strain field is then taken again, in this second step, as initial strains to the whole QD nanostructure with the capping layer in order to analyse the further induced strain due to the capping process. The boundary conditions of the pyramidal surfaces of quantum dots are now changed from the original traction-free condition, in the first step, to the continuity conditions of the appropriate displacement and stress components in the current step. Hence the stress and strain would certainly redistribute inside the nanostructure system. Again, the substrate, the wetting layer and the island are analysed together with the capping layer for electronic structures of the nanostructure system, once strain fields have been determined.

- Model III (simplified model). In this model the capping layer is excluded completely in the strain analysis, since the capping layer is assumed not to experience the induced deformation of islands during the self-assembly QDs process. The model considers only the first step of the strain analysis of the two-step model, but not the second step, i.e. the strain analysis is performed as if there were no capping layer at all in the system [9-11]. Once the strain analysis has been completed, the capping layer is then added to the system. The addition of the capping layer is only for the purpose of solving the Schrödinger equation to obtain the electronic structure of the nanostructure system. Hence the capping layer is consequently assumed to be unstrained in this model.

In this paper, two QD structures are considered in the strain analysis, namely the cases where the heights of pyramid quantum-dot island are $h=3 \mathrm{~nm}$ (structure A) and $6 \mathrm{~nm}$ (structure B), respectively. For each structure, both the cases of buried QD and the surface QD are discussed in calculations. Normal strain components $\varepsilon_{x x}$ and $\varepsilon_{z z}$ along the $z$-axis are shown in figures 2( $a$ ) and $(b)$ for structure A, respectively. It is not surprising that these strain components are discontinuous along the InAs/GaAs interface as one would expect from the continuum theory. Note that, as seen in the figure, the strain field $\varepsilon_{x x}$ inside the dot is compressive while $\varepsilon_{z z}$ is tensile. These reflect the fact that the lattice constant of InAs is larger than that of GaAs. Strain distributions obtained from model II have significant differences from the other two models. Moreover, it is found from the calculated results that the bigger the island is, the bigger the difference. This implies that the strain field inside and in the neighbourhood of the dot is sufficiently redistributed after the capping layer has been deposited.

It is of interest to point out that strain fields obtained by model III will be exactly the same as those of surface QDs, since the capping layer is excluded completely in the strain analysis. Figure 2 clearly shows that the compressive strains 


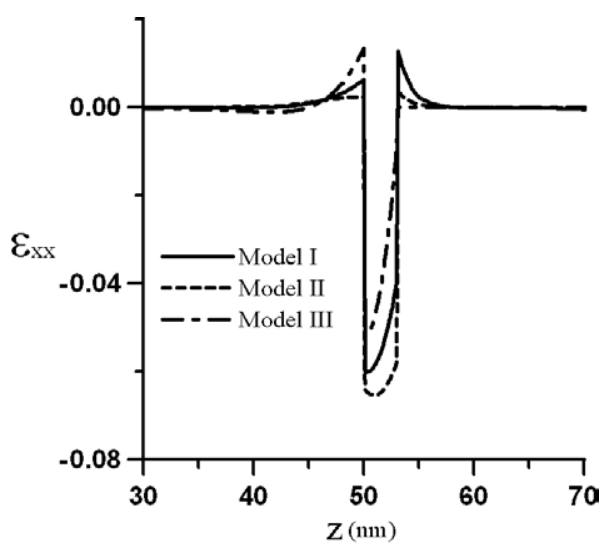

(a)

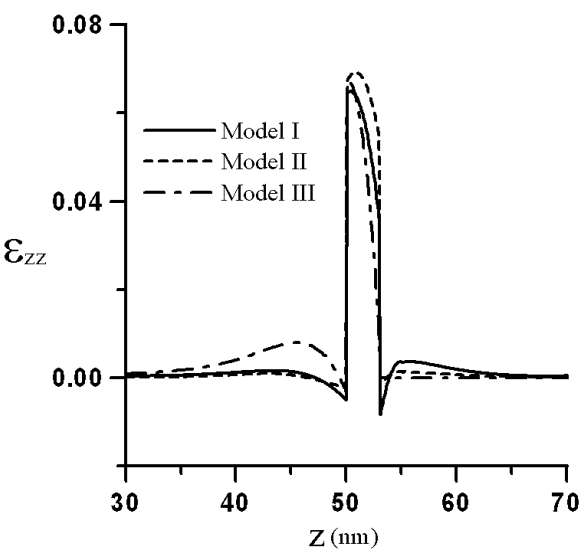

(b)

Figure 2. Strain components, $(a) \varepsilon_{\mathrm{xx}}$ and $(b) \varepsilon_{z z}$, for structure A with the capping layer plotted along the $z$-axis. The different lines correspond to the different models.

$\varepsilon_{x x}$, within the dot, obtained from model III are smaller than those from models I and II. In other words, the uncapped InAs dot will experience smaller compressive strains $\varepsilon_{x x}$ than the buried one. It is easier to explain the results by considering models I and III. InAs experiences compression from GaAs, while GaAs experiences tension from InAs, owing to mismatches of lattice constants of two materials. The buried dot (see model I) certainly experiences more compression from its surroundings, both the substrate and the capping layer, than the surface one (see model III), where it is compressed from the substrate alone as indicated in figure 2. Similarly, for the substrate near the InAs/GaAs interface, GaAs will experience tension from InAs. For the surface QDs, the tensile effects will reflect on the substrate alone, while the same effects will be shared by the substrate and the capping layer for the buried ones. Hence the tensile strains $\varepsilon_{x x}$, in the substrate and near the interface, for model III are larger than those for model I again as revealed in figure 2. Similar phenomena can also be observed for cases of another size of QD. Hence the existence of the capping layer tends to reduce the tensile $\varepsilon_{x x}$ in the substrate, while it increases the compressive $\varepsilon_{x x}$ in QD, even if the size of QD remains unaltered during the capping process.

On the other hand, in the capping layer, model I always has larger compressive or tensile strains than those from model II, as shown in figure 2. And, inside the dots, model II always has larger compressive $\varepsilon_{x x}$ and larger tensile $\varepsilon_{z z}$ than those from model I. These can be interpreted as the following: since the capping layers are added after the dots are formed in model II, the capping layers are likely to be less strained than those in model I. On the other hand, the dots deform with both the substrate and the capping layer as a single body in model I. Hence, the dots have more constraints to deform from their surroundings in this model. Consequently, model I has smaller strains than those in model II inside the dot. In summary, model I is likely to overestimate the strains in the capping layer, compared to model II, and underestimate those inside the dots.

The hydrostatic strain and biaxial strain are defined, respectively, as

$$
\varepsilon_{\mathrm{h}}=\varepsilon_{x x}+\varepsilon_{y y}+\varepsilon_{z z} \quad \text { and } \quad \varepsilon_{\mathrm{b}}=\varepsilon_{z z}-\frac{\left(\varepsilon_{x x}+\varepsilon_{y y}\right)}{2} .
$$

The strain distribution along the $z$-axis (the line through the QD) is plotted. From the calculated results, the hydrostatic strain and the biaxial strain are negative and positive, respectively, within the dot, and in fact this is a common characteristic of SAQD [18]. The barrier experiences a small strain because the GaAs barrier is stiffer than InAs dots. The strain fields obtained here will be used as an input for the confinement potentials and the electric structure calculations in the next section.

\section{Confinement potential and electronic structure}

For strained QD nanostructures, the confinement potential can be written as a sum of energy offsets of the conduction (or the valence) band, $V_{\text {band }}$, and the strain-induced potential, $V_{\text {strain }}$, as

$$
V(\mathbf{r})=V_{\text {band }}(\mathbf{r})+V_{\text {strain }}(\mathbf{r}) .
$$

The band structure contribution is determined by the difference in band gap energies of the constituent materials of the heterostructure. Since strain effects induce an extra potential field $V_{\text {strain }}$, which is determined via deformation potential theory, the alteration in the band structure and optical properties of the QD system calls for further investigation. In spite of inhomogeneous distribution of induced strain (as depicted in figure 2), the Pikus-Bir Hamiltonian [14, 19] together with the computed strain field and the LuttingerKohn Hamiltonian [4, 5] is employed as usual as the firstorder approximation to analyse strain-induced potentials in QD nanostructures. The electric material properties used here are taken from [20].

The confinement potentials in semiconductor QDs, including the strain-induced effects, are piecewise continuous functions of position. These potentials, along the $z$-axis, are shown in figure 3 for both electrons and holes for structure A with the capping layer. The energies are relative to the unstrained GaAs valence-band edge. The split-off valence band is sufficiently far off in energy from the heavyhole edge and light-hole edge, so it plays no part in our calculations [14]. It is found that the strain effects on potentials are non-negligible, especially in the quantum-dot regions. 


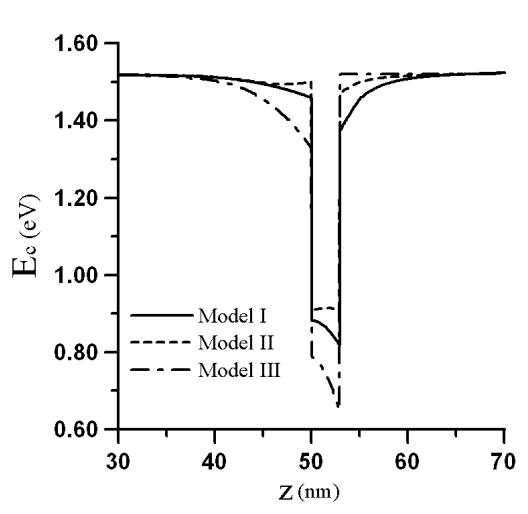

(a)

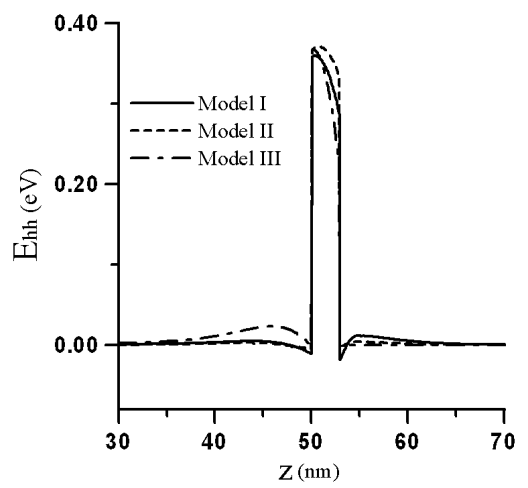

(b)

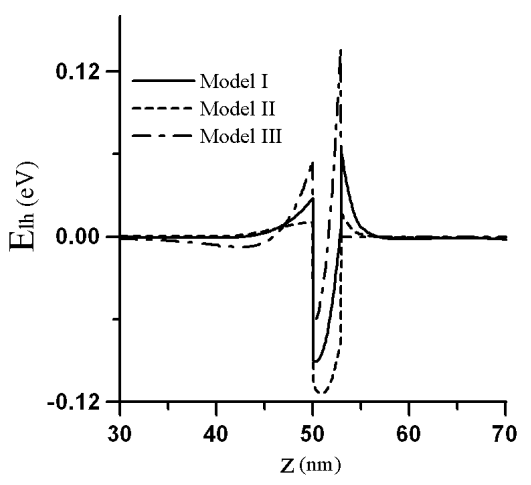

(c)

Figure 3. Energy bands of the strained InAs/GaAs QD heterostructures plotted along the $z$-axis: $(a)$ the conduction band edge; $(b)$ the heavy-hole band edge; $(c)$ the light-hole band edge.

The different lines correspond to the different models for calculation of the strain.

Due to the compressive strain $\varepsilon_{\mathrm{h}}$ inside the dot, the conduction band is reduced as in figure $3(a)$. This decrease obtained from model II is larger than those from the other two models. It is because the compressive strains $\varepsilon_{\mathrm{h}}$ calculated from model II are the largest among all the three models. The conduction band inside the dot calculated from model II is nearly a homogeneous energy. Moreover, the heavy-hole band and the light-hole band, respectively in figures $3(b)$ and $(c)$, are dependent on the magnitude and sign of the biaxial strain, $\varepsilon_{\mathrm{b}}$. The band gap of the strained InAs dot increases and the conduction band decreases. For model II, the band gap of structure $\mathrm{A}$ is increased by $142 \mathrm{meV}$ and the conduction band is decreased to about $313 \mathrm{meV}$. Similar results have been obtained by Tadic et al for the cylindrical QD [14].
Table 1. Calculated energy levels from three different models for structure A with the capping layer.

\begin{tabular}{llllll}
\hline Model & $E_{\mathrm{e} 0}(\mathrm{eV})$ & $E_{\mathrm{e} 1}, E_{\mathrm{e} 2}^{a}(\mathrm{eV})$ & $E_{\mathrm{h} 0}(\mathrm{eV})$ & $E_{\mathrm{h} 1}(\mathrm{eV})$ & $E_{\mathrm{h} 2}(\mathrm{eV})$ \\
\hline I & 1.323 & 1.479 & 0.157 & 0.052 & 0.051 \\
II & 1.354 & 1.495 & 0.170 & 0.059 & 0.058 \\
III & 1.266 & 1.447 & 0.145 & 0.042 & 0.041 \\
\hline
\end{tabular}

${ }^{\mathrm{a}} E_{\mathrm{e} 1}$ and $E_{\mathrm{e} 2}$ are degeneracies of energy levels.

Table 2. Calculated transition energies from three different models for structure A with the capping layer.

\begin{tabular}{llll}
\hline Model & $E_{\mathrm{e} 0-\mathrm{h} 0}(\mathrm{eV})$ & $E_{\mathrm{e} 1-\mathrm{h} 1}(\mathrm{eV})$ & $E_{\mathrm{e} 1-\mathrm{h} 2}(\mathrm{eV})$ \\
\hline I & 1.166 & 1.427 & 1.428 \\
II & 1.184 & 1.436 & 1.437 \\
III & 1.121 & 1.405 & 1.406 \\
\hline
\end{tabular}

The three-dimensional steady-state effective-mass Schrödinger equation is used to investigate the behaviour of individual carriers in QD nanostructures. The equation is solved numerically, in this paper, by the same finite element method package. The ground, first excited, second excited energy levels calculated from the three different models are summarized in table 1 for structure A. Due to the $C_{4 v}$ symmetry of the pyramidal dot, the first and second excited electron states, $E_{\mathrm{e} 1}$ and $E_{\mathrm{e} 2}$, are degenerate in our calculations. However, in the valence band, the first and second excited hole levels, $E_{\mathrm{h} 1}$ and $E_{\mathrm{h} 2}$, are slightly split due to mixing between bulk states.

While the corresponding transition energies between each state are shown in table 2, the transition energy is defined as the difference between the electron energy level and the hole energy level. These transition energies are related to the peaks of photoluminescence (PL) spectra. The optical conductivity has peaks at particular wavelengths of light that are more strongly absorbed. These wavelengths in turn can be expected to be the strongest emission wavelengths. The fundamental transition energy $E_{\mathrm{e} 0} \rightarrow E_{\mathrm{h} 0}$ of $1.184 \mathrm{eV}$ calculated from model II for structure $\mathrm{A}$ is in good agreement with the peak of $1.19 \mathrm{eV}$ from an experimental photoluminescence emission spectrum [21]. On the other hand, the transition energies computed from models I and III are $1.166 \mathrm{eV}$ and $1.121 \mathrm{eV}$, respectively. Therefore it seems reasonable to conclude that model II leads to the better simulation of experimental results, owing to its ability to model a real self-assembly fabrication process better than the other two models.

The fundamental transition energies of the buried and surface QDs, respectively, obtained from model II are $1.184 \mathrm{eV}$ and $0.933 \mathrm{eV}$ for structure $\mathrm{A}$, and $1.070 \mathrm{eV}$ and $0.726 \mathrm{eV}$ for structure B. The transition energy of the surface QD corresponds to a wavelength of 1329 (1708) nm for structure A (B). For the buried QD of structure A (B), the wavelength becomes 1047 (1159) nm which is corresponding to a blueshift of 282 (549) $\mathrm{nm}$ in wavelength and an energy shift of 0.251 (0.344) eV compared to that of the surface QD. This is mainly attributed to the increase of the energy gap induced by compressive strain. Phenomena of blue-shift in wavelength for buried QDs have been observed from the experimental data of photoluminescence emission [22]. In addition, the calculated results clearly indicate that the transition energy 
Two-step strain analysis of self-assembled InAs/GaAs quantum dots

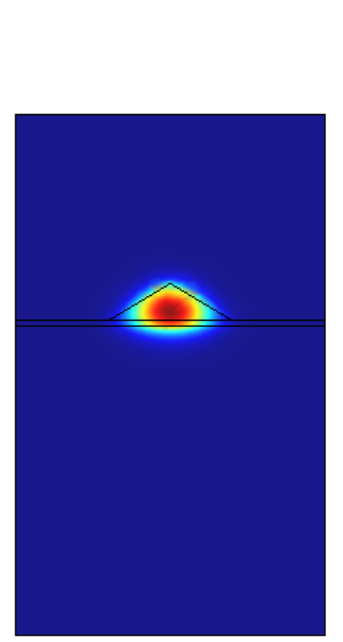

(a)

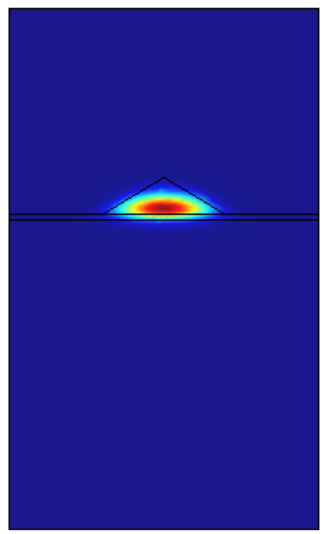

(b)

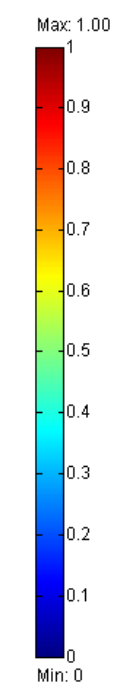

Figure 4. Probability density function profiles for energy levels of the ground state corresponding to $(a)$ electron energy and $(b)$ hole energy. The cross section is the $y z$-plane through the dot centre for structure A with the capping layer.

(This figure is in colour only in the electronic version)

becomes smaller as the dot size increases, for the buried (or surface) QDs. Similar results have also been reported by Stier et al [23].

Probability density function profiles, defined by the square of wavefunctions, $|\psi|^{2}$, for the ground state in a QD nanostructure simulated by model II, are shown in figure 4 . Figures $4(a)$ and $(b)$ are the probability density function profiles of the electron state and the hole state, respectively. These energy states are localized and have atomic-orbital-like s-type. In figure $4(a)$, the probability density distributions are confined almost to the entire quantum-dot island region. This could result due to the relatively isotropic nature of the confined potentials for electrons. On the other hand, in figure $4(b)$, the ground hole state is likely to be more confined at the bottom of the island due to the anisotropic characteristic of the confined potential of the heavy hole. In addition, there are substantial differences in the probability density distributions of electron and hole states, computed separately here, due to the differences in confinement energies and effective masses.

\section{Conclusions}

In this paper, a novel two-step model based on the theory of linear elasticity with the aid of finite element analysis has been proposed to investigate the strain field of quantum dots. The strain effects on the optoelectronic properties of the selfassembled pyramidal InAs/GaAs quantum-dot structures have also been investigated. The two-step model was proposed to accommodate the assumption that the capping layer does neither experience nor affect the induced deformation of quantum dots during the self-assembly process, since the capping layer is added after the self-assembly process of quantum dots. The proposed two-step model took into account the sequence of the fabrication process of quantum dots. In the first step, the strain field in the heterostructure system without the capping layer was first analysed, where the mismatch of lattice constants between the wetting layer and the substrate was the driving source and was treated as initial strain in the analysis. The obtained strain field was then treated as initial strain for the complete heterostructure system, with the capping layer, in the second step. The changes of the boundary conditions of the pyramidal surface, from traction-free to the continuity conditions of the appropriate displacement and stress components, served as the other driving source of strain in this step. The numerical results of the final strain field from this new model have shown significant difference from the other two models where the sequence of the fabrication process was neglected.

The calculated strain field from the two-step analysis has also been used as an input for the electronic band structure calculation. Though the strain distribution in the quantumdot nanostructures is not uniform, the Pikus-Bir Hamiltonian and the Luttinger-Kohn Hamiltonian have, nevertheless, been employed as an approximation to analyse strain-induced potentials in the quantum-dot nanostructures. The energy levels and the wavefunction distribution have been obtained by solving the three-dimensional steady-state effective-mass Schrödinger equation, including the strain-induced potential. The wavelengths based on the strain fields from the twostep analysis differed substantially from their counterparts in the conventional strain analysis model. The calculated optical wavelength from this new model agrees with previous experimental photoluminescence studies. In summary, it seems reasonable to conclude that the novel two-step model leads to the better simulation of experimental results, owing to its ability to model a real self-assembly fabrication process better than the other two models. Hence, the proposed twostep strain analysis is crucial for future optical analysis and applications.

\section{Acknowledgments}

This work is carried out in the course of research sponsored by the National Science Council of Taiwan under grants NSC922212-E-002-072 and NSC93-2212-E-002-015.

\section{References}

[1] Bimberg D, Grundmann M and Ledentsov N N 1999 Quantum Dot Heterostructures (West Sussex: Wiley)

[2] Harrison P 2000 Quantum Wells, Wires and Dots: Theoretical and Computational Physics (West Sussex: Wiley)

[3] Chakraborty T 1999 Quantum Dots: A Survey of the Properties of Artificial Atoms (Amsterdam: Elsevier)

[4] Pikus G E and Bir G L 1974 Symmetry and Strain-Induced Effects in Semiconductors (New York: Wiley)

[5] Shchukin V A, Bimberg D, Malyshkin V G and Ledentsov N N 1998 Phys. Rev. B 5712262

[6] Andreev D, Downes J R, Faux D A and O'Reilly E P 1999 J. Appl. Phys. 86297

[7] Benabbas T, Androussi Y and Lefebvre A 1999 J. Appl. Phys. 861945

[8] Muralidharan G 2000 Japan. J. Appl. Phys. 39 L658

[9] Johnson H T, Nguyen V and Bower A F 2002 J. Appl. Phys. 924653

[10] Freund L B and Johnson H T 2001 J. Mech. Phys. Solids 491925

[11] Johnson H T, Freund L B, Akyüz C D and Zaslavsky A 1998 J. Appl. Phys. 843714 
[12] Kuo M K, Lin T R, Liao B T and Yu C H 2005 Physica E 26199

[13] Lin T R, Kuo M K, Liao B T and Hung K P 2004 Bull. Coll. Eng. NTU 913

[14] Tadic M, Peeters F M, Janssens K L, Korkusinski M and Hawrylak P 2002 J. Appl. Phys. 925819

[15] Pryor C, Kim J, Wang L W, Williamson A J and Zunger A 1998 J. Appl. Phys. 832548

[16] Freund L B 2000 Int. J. Solids Struct. 37185

[17] Chuang S L 1995 Physics of Optoelectronic Devices (New York: Wiley)
[18] Saito T, Nakaoka T, Kakitsuka T, Yoshikuni Y and Arakawa Y 2005 Physica E 26217

[19] Chao C Y P and Chuang S L 1992 Phys. Rev. B 464110

[20] Levinshtein M, Rumyantsev S and Shur M 1996 Handbook Series on Semiconductor Parameters (Singapore: World Scientific)

[21] Pal D, Stoleru V G, Towe E and Firsov D 2002 Japan. J. Appl. Phys. 41482

[22] Saito H, Nishi K and Sugou S 1998 Appl. Phys. Lett. 732742

[23] Stier O, Grundmann M and Bimberg D 1999 Phys. Rev. B 595688 